\title{
Synthesis and in situ mechanism of nuclei growth of layered double hydroxides
}

\author{
H S PANDA, R SRIVASTAVA ${ }^{\dagger}$ and D BAHADUR* \\ Department of Metallurgical Engineering and Materials Science, ${ }^{\dagger}$ School of Biosciences and Bioengineering, \\ Indian Institute of Technology Bombay, Mumbai 400 076, India
}

MS received 6 December 2009

\begin{abstract}
A host-guest material such as layered double hydroxide (LDH) has generated immense interest in current research due to its technological importance, whereby its dimension significantly affect its mechanical and other physical properties. The purpose of this study was to prepare $\mathrm{Mg} / \mathrm{Al}-\mathrm{LDHs}$ by systematically varying the molar concentration of cations, aging time and $\mathrm{pH}$. The prepared LDHs were characterized by powder $\mathrm{X}$-ray diffraction, Fourier transform infrared spectroscopy, thermal analysis and transmission electron microscopy to confirm their formation and morphology. We qualitatively observed a new growth route for LDH system which is dissimilar to the existing growth mechanism. The rate of growth is shown to be slower than the well known Ostwald ripening process. This unusual behaviour is due to the formation of effective passivation layer by $\mathrm{Na}^{+}$ions around the generated LDHs nuclei. This suggested growth mechanism will be helpful in further controlling the particle size of other LDH, which may be useful for various future applications.
\end{abstract}

Keywords. LDHs; growth mechanism; morphology.

\section{Introduction}

Over the past decade, layered double hydroxides (LDHs), otherwise named as hydrotalcites have attracted attention of the researchers due to their layered structure and their high anion exchange capacity, which make them potential candidature for technical applications in various domains, such as medicine, catalysis, separation technology, polymer reinforcement, electrochemistry, etc. (Adams 1987; Fogg et al 1999; Khan et al 2001; Kwak et al 2004; Li et al 2005a; Zhang et al 2005; Panda et al 2009). The structure of LDHs can be described from brucite-type $\mathrm{Mg}(\mathrm{OH})_{2}$, where metal ions are situated at the centre of the octahedron and the vertices are occupied by hydroxide ions. The positive charge is generated by partial substitution of divalent metal cations by trivalent ones within the brucite-like layers and is balanced by an equal negative charge from the inter layer solvated anions (Lakraimi et al 2000). The general formula for $\mathrm{LDH}$ is $\mathrm{M}^{\mathrm{II}}{ }_{1-x} \mathrm{M}^{\mathrm{III}}{ }_{x}$ $(\mathrm{OH})_{2}\left(\mathrm{Y}^{n-}\right)_{x / n} \cdot \mathrm{mH}_{2} \mathrm{O}$, where $x=0 \cdot 2-0 \cdot 33, m=1-3 \mathrm{Y} / 2$, $\mathrm{M}^{\mathrm{II}}=$ divalent cations, $\mathrm{M}^{\mathrm{III}}=$ trivalent cations and $\mathrm{Y}^{n-}=$ $n$-valent anions (Kaneyoshi and Jones 1999). A large number of such compounds are known, and one of their important features is the relative ease of synthesis of a range of $\mathrm{LDH}$ matrices with a significant variation of layer charge, the concentration of the reactants in the reaction

\footnotetext{
*Author for correspondence (dhirenb@iitb.ac.in)
}

medium and the synthesis procedures. The influence of the $\mathrm{M}^{\mathrm{II}}$ and $\mathrm{M}^{\mathrm{III}}$ salts, the $\mathrm{M}^{\mathrm{II}} / \mathrm{M}^{\mathrm{III}}$ ratio and the interlayer anion on the crystallinity and morphology of the co-precipitated LDHs have been widely studied in the literature cited previously (Franklin et al 1995; Chang et al 2005).

Controlling the particle size of LDHs is a challenge, as it is important for many potential applications. For example, nanodimension LDHs particles can be used as additives in agricultural plastic film since their infrared absorbing properties enhance the heat retaining properties of the film (Hancock 1990; Adachi-Pagano et al 2003). Also, the dimension of the particle plays a significant role in retaining the mechanical and other physical properties of polymers. Aluminum-substituted LDHs compounds are important because of their stability and high electrochemical performance. The effect of electrochemical behaviour on crystallite size of a nickel-metal hydride electrode was analysed by mathematical model, which clearly showed that the crystallite size significantly affects the electrode performance. Recent experimental findings (Hu and Lei 2007) show that the crystallite size of the sample has evident effects on its discharge capacity at high current density. Several articles (Ennadi et al 2000; Prevot et al 2001; We et al 2005; Panda et al 2008b, 2009) have been published so far on LDHs containing intercalated anion. But the lack of literature on the growth parameters of LDHs during synthesis and its conviction that the growth of nano LDHs occurs via the Ostwald ripening behaviour, despite experimental verifi- 
cation of different constraints used in synthesis, are absent. Different synthesis techniques have been developed so far to control the particle size, but the co-precipitation route is one of the most preferred routes to synthesize $\mathrm{M}^{\mathrm{II}} / \mathrm{M}^{\mathrm{III}}$ LDHs. Recent theoretical investigation (Talapin et al 2001) on the growth of nanocrystals suggest that the growth could be either controlled by diffusion of the particles, i.e. Ostwald ripening, or controlled by the reaction at the surface, or by both parameters even in the absence of capping agent. In these cases the nano LDHs growth after nucleation in absence of any capping agent is the most logical ground for inquiring the growth kinetics.

In this work we have synthesized $\mathrm{Mg} / \mathrm{Al}-\mathrm{CO}_{3} \mathrm{LDHs}$ systems by varying the molar concentration of metal ions. For a particular metal ion concentration, we qualitatively investigated the growth behaviour of nanocrystals upon varying the synthesis parameters. In our studies we noticed some novel features; the growth of LDHs crystals can be strongly affected by the reactant itself and the growth model (figure 1) is qualitatively different compared to that of the universally accepted Ostwald ripening process. To investigate the in situ particles growth, there are no suitable techniques available, although transmission electron microscope (TEM) is the most suitable technique to determine the average particle size and size distribution ex situ, but due to high agglomeration tendency of LDHs particles, it is restricted to observe individual particles during measurement and it may therefore agglomerate or fold the layered platelets. In this context, the average crystallite size was calculated from background subtracted X-ray diffraction (XRD) patterns by using Scherrer formula. The FWHM was calculated by fitting Gaussian on the XRD patterns through Origin software. Finally, the morphology of LDHs particles was compared using TEM data.

\section{Experimental}

Magnesium nitrate hexahydrate was obtained from Fluka with $99 \%$ purity. Aluminium nitrate nonahydrate (98\%),

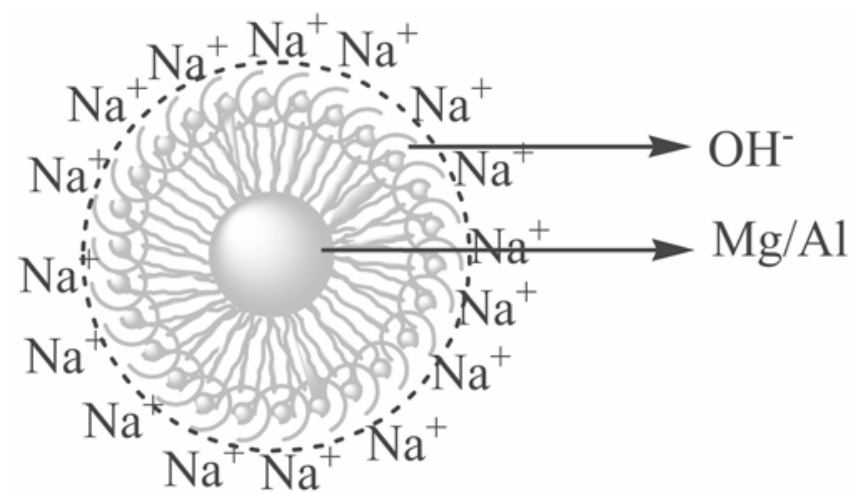

Figure 1. Schematic representation of in situ $\mathrm{Na}^{+}$passivation layer around the LDHs nuclei. sodium carbonate $(99 \%)$ and sodium hydroxide were obtained from Thomas Beaker Chemicals Limited. LDHs having carbonate as interlayer anion with $\mathrm{Mg}$ and $\mathrm{Al}$ as the octahedral cations (designed $\mathrm{MgAl}-\mathrm{CO}_{3}$ ) were prepared using an established method (Whilton et al 1997). Salt solutions with molar ratios of $2: 1,3: 1$ and $4: 1$ were prepared by adding $\mathrm{Mg}\left(\mathrm{NO}_{3}\right)_{2} \cdot 6 \mathrm{H}_{2} \mathrm{O}(2 \cdot 2,3 \cdot 3$ and $4.4 \mathrm{mmol})$ and $\mathrm{Al}\left(\mathrm{NO}_{3}\right)_{3} \cdot 9 \mathrm{H}_{2} \mathrm{O}(1.1 \mathrm{mmol})$ in $18 \mathrm{ml}$ de-ionized water. After complete dissolution, the above solution was added drop wise to a vigorously stirred $\mathrm{Na}_{2} \mathrm{CO}_{3}$ solution in $60 \mathrm{ml}$ de-ionized water. Freshly prepared $1 \mathrm{M} \mathrm{NaOH}$ solution was then added drop wise to the above solution to maintain constant $\mathrm{pH}(\sim 11)$. The resultant gel was aged by stirring at $65^{\circ} \mathrm{C}$ for $12 \mathrm{~h}$. The precipitate was separated from the solution by centrifugation, washed several times with de-ionized water and dried in vacuum. All these reactions were carried out in an inert atmosphere.

In order to study the effect of synthesis parameters, initially the synthesis process was repeated for $\mathrm{pH} \mathrm{9,} \mathrm{pH}$ 10 and $\mathrm{pH} 11$ keeping all other parameters constant, while at $\mathrm{pH} 11$, the above reactions are studied at different aging time. Additionally, for investigating the effect of sodium hydroxide concentration on growth at particular $\mathrm{pH}$, aging time, aging temperature and metal ion concentration, particles are nucleated in various molar concentration of $\mathrm{NaOH}$. Powder X-ray diffraction (PXRD) data was collected on a Phillips PW 3040/60 diffractometer using $\mathrm{Cu}-\mathrm{K} \alpha$ radiation $(\lambda=1.5406 \AA)$ with scan step time $0 \cdot 017 \mathrm{~s}$. All measurements were made using a generator voltage $40 \mathrm{kV}$ and emission current of $30 \mathrm{~mA}$. The characteristic bonding nature between $\mathrm{CO}_{3}^{2-}$ and metal hydroxides was confirmed through Fourier transform infrared spectra (FT-IR) and back up of the layered structure was observed through thermal analysis (SDT Q600). TEM images were taken on a Phillips-CM200 electron microscope operated at $200 \mathrm{kV}$ wherein one drop of suspended samples (solvent-ethanol) was deposited on carbon coated copper grid and dried and images obtained.

\section{Results and discussion}

X-ray diffraction patterns presented in figure 2 show that well-crystallized hydrotalcite-like compounds are obtained and no other phase is identified. The X-ray diffraction for these samples typically consist of anionic clay having molecular formula $\mathrm{Mg}_{6} \mathrm{Al}_{2}(\mathrm{OH})_{16} \mathrm{CO}_{3} \cdot 4 \mathrm{H}_{2} \mathrm{O}$ with carbonate in the interlayer space. The sharp peak obtained at $d=7.69 \AA\left(2 \theta=11 \cdot 5^{\circ}\right)$ can be ascribed to $(003)(h k l)$ of a hydrotalcite structure with rhombohedral $3 \mathrm{R}$ packing of the layers. Upon considering the thickness of brucite layer, i.e. $4 \cdot 8 \AA$ (Whilton et al 1997), the gallery spacings are approximately $2 \cdot 89,3.06$ and $3.16 \AA$ for $2: 1,3: 1$ and 4:1 molar ratio, respectively. The interlayer space 
calculated is $2.89 \AA$, indicating that carbonate anions are located with their molecular plane parallel to the brucitelike layers (Zhao et al 2002). Assuming the 3R packing of the layers, lattice parameter $c$ corresponds to three times the value of $d(003)$, i.e. $c=23.07 \AA$ and this is the distance between the center of one layer to the center of other layer. Lattice parameter $a$ corresponds to the average closest metal-metal distance within a layer, and is calculated as twice the position of the diffraction maximum due to planes (110), i.e. $a=3 \cdot 04 \AA$ for $\mathrm{Mg}$ : Al ratio of $2: 1$. Further, it increases with the increasing $\mathrm{Mg}$ content on the brucite-like layers, as the ionic radius for $\mathrm{Mg}^{2+}(0.065 \mathrm{~nm})$ is larger than that for $\mathrm{Al}^{3+}(0.050 \mathrm{~nm})$. The average crystallite size in the $a$ and $c$ directions may be calculated from the values of the FWHM of the (110) and (003) diffraction peaks, respectively, and by using the Scherrer formula, i.e. $L=0 \cdot 89 \lambda /(\beta(\theta) \times \cos \theta)$, where $L$ is the crystallite size, $\lambda$ the wavelength of the radiation used, $\theta$ is the Bragg diffraction angle and $\beta(\theta)$ the FWHM (Li et al 2005b). In order to gain information on the mechanism of growth process, we plotted the crystallite size of the nano LDHs as a function of aging time at fixed temperature of $338 \mathrm{~K}, \mathrm{pH}(11)$ and $\mathrm{NaOH}$ concentration $(1 \mathrm{M})$ in figures 3 and 4 . It is observed from this figure that the size of the growing nuclei depends strongly on the reaction time, which is the expected behaviour within the diffusion-limited Ostwald ripening process. To gain further insight into the mechanism of growth, we observed the crystallite size as a function of $\mathrm{pH}$ of the reaction medium during synthesis at fixed temperature $(338 \mathrm{~K})$, aging time $(12 \mathrm{~h})$ and sodium hydroxide concentration (1M). It is evident from this study that the growth of crystallite size at a specific time depends

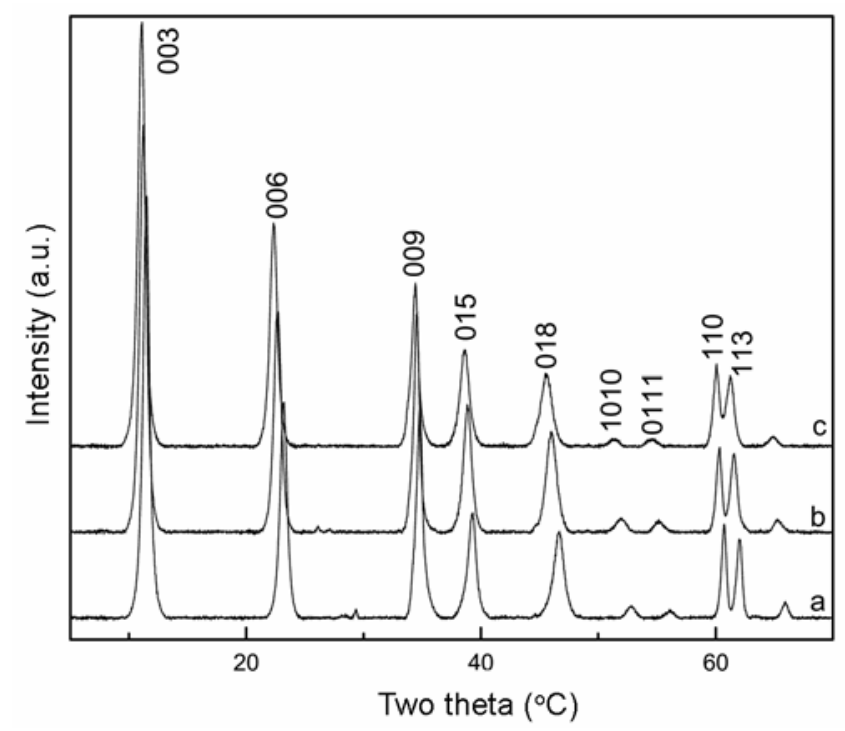

Figure 2. XRD patterns of $\mathrm{Mg} / \mathrm{Al}-\mathrm{CO}_{3} \mathrm{LDHs}$ having molar ratios: a. $\mathrm{Mg} / \mathrm{Al}-2: 1$ (12 h), b. Mg/Al-3 : 1 (12 h) and c. Mg/Al$4: 1(12 \mathrm{~h})$. on the reaction medium $\mathrm{pH}$, where $\mathrm{NaOH}$ is the prime parameter to maintain the required $\mathrm{pH}$. This can be explained by considering the hydroxyl ions in the solution, which promote the Ostwald ripening factor. As for higher $\mathrm{pH}$, in the beginning sodium hydroxide solution was added in sodium carbonate solution to attain the required $\mathrm{pH}$.

We have probed this unusual behaviour by carrying out different studies, systematically varying the sodium hydroxide concentration at different $\mathrm{pH}$ and keeping all other parameters constant (figure 5). Interestingly, we found that (table 1) the crystallites growth decreases with decreasing sodium hydroxide concentration. Additionally,

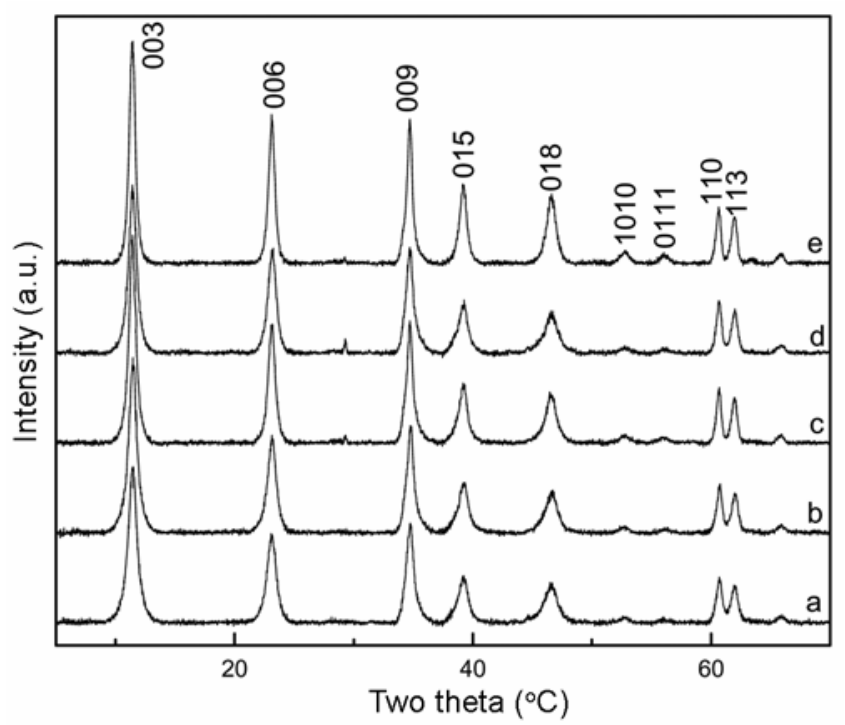

Figure 3. $\mathrm{XRD}$ patterns of $\mathrm{Mg} / \mathrm{Al}-\mathrm{CO}_{3} \mathrm{LDHs}$ with constant temperature $\left(65^{\circ} \mathrm{C}\right)$ having aging time: a. $2 \mathrm{~h}$, b. $6 \mathrm{~h}, \mathrm{c} .12 \mathrm{~h}, \mathrm{~d}$. $18 \mathrm{~h}$ and e. $24 \mathrm{~h}$.

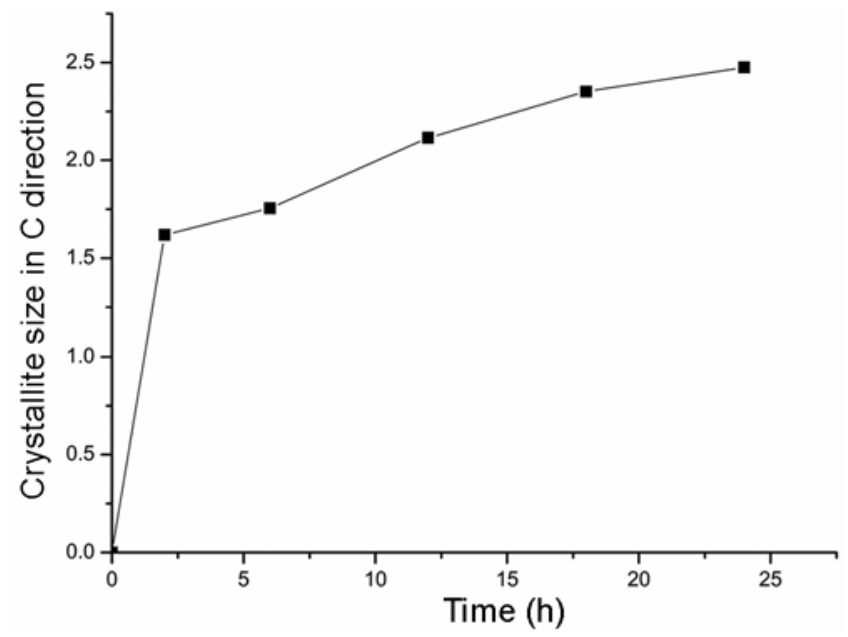

Figure 4. Variation of crystallite size as a function of aging time of $\mathrm{Mg} / \mathrm{Al}-\mathrm{CO}_{3} \mathrm{LDHs}$. 

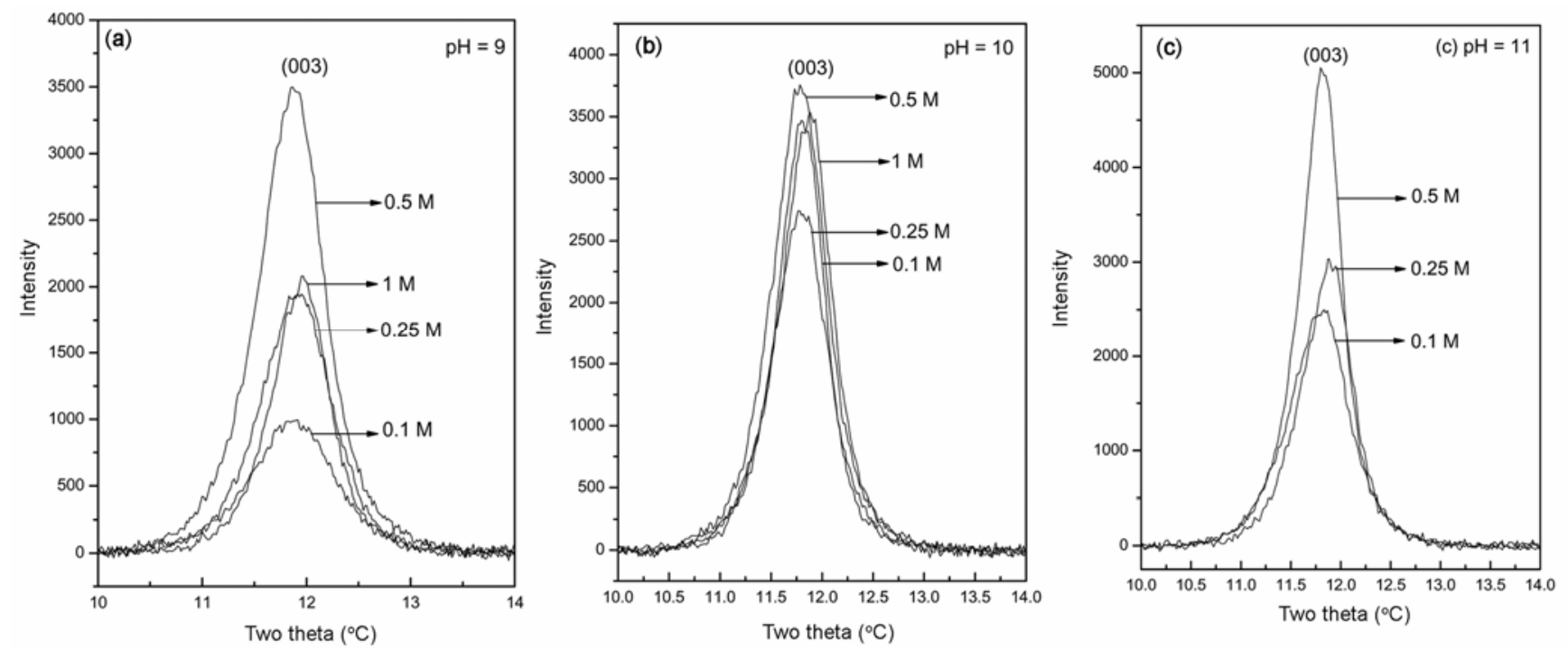

Figure 5. XRD patterns of $\mathrm{Mg} / \mathrm{Al}-\mathrm{CO}_{3} \mathrm{LDHs}$ at $\mathrm{pH}$ : (a) 9, (b) 10 and (c) 11 having different molar concentration of $\mathrm{NaOH}$.

Table 1. Variation of crystallite size of $\mathrm{Mg} / \mathrm{Al}-\mathrm{CO}_{3} \mathrm{LDHs}$ having $\mathrm{Mg} / \mathrm{Al}$ molar ratios 2.

\begin{tabular}{|c|c|c|c|}
\hline \multirow{2}{*}{$\begin{array}{l}\text { Molar concentration } \\
\text { of } \mathrm{NaOH}(\mathrm{M})\end{array}$} & \multicolumn{3}{|c|}{ Average crystallite size } \\
\hline & pH 9 & $\mathrm{pH} 10$ & pH 11 \\
\hline 1 & $2 \cdot 42$ & $2 \cdot 57$ & - \\
\hline $0 \cdot 5$ & $2 \cdot 08$ & $2 \cdot 45$ & $3 \cdot 1$ \\
\hline $0 \cdot 25$ & $1 \cdot 65$ & $2 \cdot 43$ & $2 \cdot 59$ \\
\hline $0 \cdot 1$ & 1.97 & $2 \cdot 85$ & $2 \cdot 24$ \\
\hline
\end{tabular}

we observe formation of nano LDHs in all the reactions is instantaneous and only the growth of the crystallites is affected by the presence of $\mathrm{NaOH}$. As the growth rate was found to depend strongly on the amount of water present, the possibility of growth was excluded due to initial water taken for preparing sodium carbonate and salt solution, as it was same for all reaction medium. The initial decrease followed by a slow increase at higher concentration of $\mathrm{NaOH}$ suggests that the addition of base not only maintains the $\mathrm{pH}$ of the reaction medium but also supports the nuclei growth. Hence, the results in figure 5 point to a completion of two reaction parameters, where one parameter suppresses the other at a critical limit. It is important to note that for $\mathrm{LDH}$, nucleation and growth are assisted by water. But, at constant water concentration, upon increasing the concentration of $\mathrm{NaOH}$ above a critical limit, it promotes the nuclei to grow. In order to get conformation of our hypothesis, the above process was repeated for other pHs keeping all the parameters constant (figures $5 \mathrm{~b}$ and $\mathrm{c}$ ). The same trend was observed in all the cases, indicating that hydroxyl or sodium ions of sodium hydroxide play a key role to control the particle size. In order to identify if hydroxyl or sodium or both ions take part in the growth process, we have made a logical argument. As we go on reducing the sodium hydroxide concentration, the overall solution concentration increases, as more amount of sodium hydroxide solution is required to maintain constant $\mathrm{pH}$ for a particular salt concentration. Such a behaviour is unusual, since one does not expect this retardation of growth process, as small amount of water (Viswanatha et al 2004) specifically increases the size of crystallites. This behaviour is observed below the critical concentration of $\mathrm{NaOH}(0.25 \mathrm{M})$. This suppression of crystal growth can be explained by using recently reported capping mechanism for zinc oxide nanocrystals (Viswanatha et al 2007), which is the reverse of the Ostwald ripening phenomenon. In the presence of sodium hydroxide, sodium ions adjoining the generated nuclei act as a capping agent and hinder the nucleating particles growth. As the hydroxyl ion attaches to the surface of the nano LDHs, it is noticeable that the sodium ion acts as a counter ion and hinders the other nucleating metal ion. From the table, it was noticed that at higher $\mathrm{pH}$ the critical limit point shifts to further lower concentration of sodium hydroxide. This might be due to the presence of excess sodium ions.

In order to further confirm the effect of sodium ion concentration on particles morphology, LDHs particles were characterized using TEM and it was observed that there is significant change of morphology at $\mathrm{pH} 9$ with 


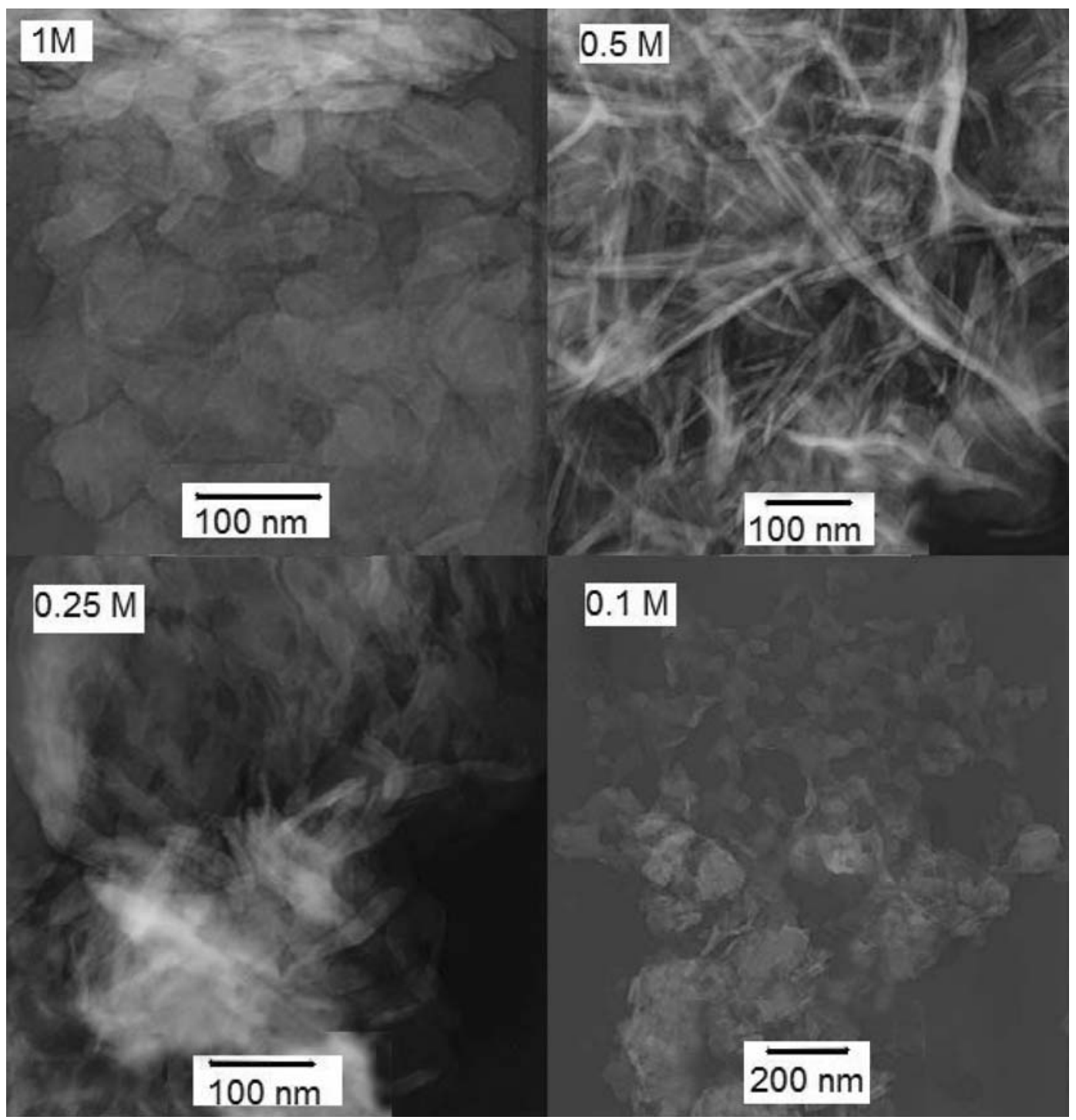

Figure 6. TEM micrographs of $\mathrm{Mg} / \mathrm{Al}-\mathrm{CO}_{3} \mathrm{LDHs}$ prepared at $\mathrm{pH} 9$ having different molar concentration of $\mathrm{NaOH}$.

varying molar concentration of $\mathrm{NaOH}$. The unusual morphology (figure 6) of LDHs particles was supported the above discussion. However, the exact reason is still unclear and this may be due to the effect of base concentration on the solid solubility of metal hydroxides. Again, in some cases, LDHs platelets tend to form nanorods due to the bigger size/less force in between the platelets and the mechanism was explained in our previous report (Panda et al 2008).

Finally, in infrared spectra (figure not provided), the broad peak found at around $3443 \mathrm{~cm}^{-1}$ was due to stretching of $\mathrm{O}-\mathrm{H}$ groups and the bands around $672 \mathrm{~cm}^{-1}$ was attributed to the $\mathrm{M}-\mathrm{O}$ stretching vibration, which remain unchanged for all the cases. Thermal analysis (TGA and DTA) curves for the LDHs prepared in all molar concentration of $\mathrm{NaOH}$ provided usual thermal degradation behaviour, i.e. $50-160^{\circ} \mathrm{C}, 170-300^{\circ} \mathrm{C}$ and $310-450^{\circ} \mathrm{C}$, due to loss of water molecules, decomposition of interlayer anions and dehydroxylation of hydroxyl groups which are attached to the metal ions respectively. These results confirmed that the existence of layered structure and $\mathrm{NaOH}$ concentration do not affect the internal arrangement of atoms or ions, but significantly influence particle size and morphology.

\section{Conclusions}

We have prepared the $\mathrm{Mg} / \mathrm{Al} \mathrm{LDHs}$ via the usual coprecipitation method by using sodium hydroxide and the structure was confirmed by using powder X-ray diffraction. Growth study was performed by varying the aging time and $\mathrm{pH}$, and the growth behaviour is qualitatively similar as expected for the Ostwald ripening phenomenon. 
In earlier work, sodium hydroxide was used during synthesis for maintaining the $\mathrm{pH}$ of the reaction medium. But it has been observed that use of sodium hydroxide significantly lowers the rate of growth, which is qualitatively dissimilar than the well-known growth mechanism. This unexpected behaviour was observed for different $\mathrm{pH}$ and it occurs due to the formation of capping layer by $\mathrm{Na}^{+}$ions. Finally, this new understanding of the growth process of nano LDHs may be useful for preparing $\mathrm{LDH}$, which may be useful for various future applications.

\section{Acknowledgements}

We thank the NanoMission of Department of Science and Technology, India, for supporting this work.

\section{References}

Adams J M 1987 Appl. Clay. Sci. 2309

Adachi-Pagano M, Forano C and Besse J P 2003 J. Mater. Chem. 131998

Chang Z, Evans D G, Duan X, Vial C, Ghanbaja J, Prevot V, Roy M and Forano C 2005 J. Solid State Chem. 1782766

Ennadi A, Legrouri A, Roy A and Besse J P 2000 J. Mater. Chem. 102337

Fogg A M, Green V M, Harvey H G and O'Hare D 1999 Adv. Mater. 111466

Franklin K R, Lee E and Nunn C C 1995 J. Mater. Chem. 5 565

Hancock M 1990 Plasticulture 791439

Hu M and Lei L 2007 J. Solid State Electrochem. 11847
Kwak S, Kriven W M, Wallig M A and Choy J 2004 Biomaterials 255995

Khan A I, Lei L, Norquist A J and O'Hare D 2001 Chem. Commun. 2342

Kaneyoshi M and Jones W 1999 J. Mater. Chem. 9805

Lakraimi M, Legrouri A, Barroug A, Roy A de and Besse J P 2000 J. Mater. Chem. 101007

Li F, Wang Y, Yang Q, Evans D G, Forano C and Duan X 2005a J. Hazardous Mater. B125 89

Li F, Liu X, Yang Q, Liu J, Evans D G and Duan X 2005b Mater. Res. Bull. 401244

Panda H S, Srivastava R and Bahadur D 2008a J. Nanosci. Nanotechnol. 84218

Panda H S, Srivastava R and Bahadur D 2008b Mater. Res. Bull. 431448

Panda H S, Srivastava R and Bahadur D 2009a J. Phys. Chem. B113 15090

Panda H S, Srivastava R and Bahadur D 2009b J. Phys. Chem. C1139560

Prevot V, Casal B and Ruiz-Hitzky E 2001 J. Mater. Chem. 11 554

Talapin D V, Rogach A L, Haase M and Weller H 2001 J. Phys. Chem. B105 12278

Viswanatha R, Sapra S, Sengupta S, Satpati B, Satym P V, Dev B N and Sarma D D 2004 J. Phys. Chem. B108 6303

Viswanatha R, Amenitsch H and Sarma D D 2007 J. Am. Chem. Soc. 1294470

We Q, Olafsen A, Vistad Q B, Roots J and Norby P 2005 J. Mater. Chem. 154695

Whilton N T, Vickers P J and Mann S 1997 J. Mater. Chem. 7 1623

Zhang H, Zou K, Sun H and Duan X 2005 J. Solid State Chem. 1783485

Zhao Y, Li F, Zhang R, Evans D G and Duan X 2002 Chem. Mater. 144286 